\title{
ISOMORPHISM TYPES OF INDEX SETS OF PARTIAL RECURSIVE FUNCTIONS
}

\author{
LOUISE HAY
}

1. Let $\left\{q_{0}, q_{1}, q_{2}, \cdots\right\}$ be a Kleene enumeration of partial recursive functions. If $f$ is such a function, denote by $\theta f$ its index set, $\theta f=\left\{n \mid q_{n} \simeq f\right\}$. Insofar as the indices of a partial recursive function correspond to the different sets of "instructions" for computing its values, it is natural to ask how much of the "complexity" of the function is reflected by its index set; for example, one might expect the index set of a constant total function to differ in a fundamental way from that of a function whose domain and range are nonrecursive sets. More precisely, since the basic equivalence relation of recursion theory is recursive isomorphism (i.e., equivalence under a recursive permutation of the nonnegative integers) the following question arises: How many distinct recursive isomorphism types of index sets are there, and which properties of the corresponding functions can be used to characterize these types? This is answered in the theorem below. That the answer is independent of any particular enumeration follows from the fact, proved by Rogers in [5], that different "standard-type" enumerations are related by means of recursive permutations.

In the following, function will mean partial recursive function and degree will mean Turing degree of unsolvability. We write $\alpha R_{1} \beta$ for $\alpha$ is 1-1 reducible to $\beta$ and $\alpha \cong \beta$ for $\alpha$ is recursively isomorphic to $\beta$. The notation is that of [3], but the technique will be informal in character.

THEOREM 1. There are exactly three isomorphism types of index sets of partial recursive functions, and the type of $\theta f$ is uniquely determined by whether the domain of $f$ is null, finite or infinite. In the first two cases, $\theta f$ has degree $\mathbf{0}^{\prime}$, in the last case degree $\mathbf{0}^{\prime \prime}$.

The proof follows from several lemmas.

Lemma 1. Let $f, g$ be finite, nonvoid functions. Then $\theta f \cong \theta g$.

Proof. Assume $f=\left\{\left(a_{0}, b_{0}\right), \cdots,\left(a_{p}, b_{p}\right)\right\}$ and $g=\left\{\left(x_{0}, y_{0}\right), \cdots\right.$, $\left.\left(x_{m}, y_{m}\right)\right\}$. Let $x_{m+1}$ be distinct from $x_{0}, \cdots, x_{m}$. Using standard techniques, define a 1-1 recursive function $h$ by

$$
q_{h(n)}\left(x_{i}\right)=y_{i} \text { for } 0 \leqq i \leqq m \text { if } q_{n}\left(a_{j}\right)=b_{j} \text { for } 0 \leqq j \leqq p,
$$

Received by the editors March 25, 1965. 
$q_{h(n)}\left(x_{m+1}\right)=0$ if $q_{n}\left(a_{j}\right)=b_{j}$ for $0 \leqq j \leqq p$ and $q_{n}(x)$ is defined for some $x \in\left\{a_{0}, \cdots, a_{p}\right\}$,

$q_{h(n)}(x)$ undefined otherwise.

(We note here for future reference that for all $n$, either $q_{h(n)}=\varnothing \subseteq g$ or $q_{h(n)}$ extends $g$.)

Then

$$
\begin{aligned}
h(n) \in \theta g & \leftrightarrow q_{h(n)}\left(x_{i}\right)=y_{i} \text { for } 0 \leqq i \leqq m \text { and } q_{h(n)}(x) \text { is undefined } \\
\text { for all } x \notin\left\{x_{0}, \cdots, x_{m}\right\} & \leftrightarrow q_{n}\left(a_{j}\right)=b_{j} \text { for } 0 \leqq j \leqq p \text { and } q_{n}(x) \text { is undefined for all } \\
x \notin\left\{a_{0}, \cdots, a_{p}\right\} & \\
\leftrightarrow n \in \theta f . &
\end{aligned}
$$

So $\theta f$ is $1-1$ reducible to $\theta g$. By symmetry, $\theta g R_{1} \theta f$, which by Myhill's theorem [4] implies $\theta f \cong \theta g$.

Lemma 2. Assume $q_{e}$ has an infinite domain, and let $\alpha$ be any set whose defining predicate can be written in $A E$ form in the arithmetic hierarchy. Then $\alpha$ is 1-1 reducible to $\theta q_{e}$.

Proof. This was shown for the case where $q_{e}$ is total by Shapiro in [6]. His argument easily generalizes to functions with arbitrary infinite domains, as follows: Assume that $n \in \alpha \equiv(z)(E y) R(n, z, y)$ for a recursive $R$. Now define a recursive function $d$ by

$q_{d(n)}(z)=$ the $z$ th number $x$ for which $q_{n}(x)$ is computed in some simultaneous computation of all values of $q_{n}$.

Then $q_{d(e)}$ is clearly total if domain $q_{e}$ is infinite, and range $q_{d(e)}$ $=$ domain $q_{e}$. Now define a 1-1 recursive function $h$ by

$$
\begin{aligned}
& q_{h(n)}(x)=q_{e}(x) \quad \text { if }(E y)(E z)\left(x=q_{d(e)}(z) \wedge R(n, z, y)\right), \\
& q_{h(n)}(x) \text { undefined otherwise. }
\end{aligned}
$$

(We again note, for future reference, that $q_{e}$ extends $q_{h(n)}$ for each $n$.) Then

$$
\begin{aligned}
n \in \alpha \leftrightarrow & (z)(E y) R(n, z, y) \leftrightarrow \text { domain } q_{h(n)} \subseteq \text { domain } q_{e} \text { and } \\
& (x)\left[x \in \text { domain } q_{e} \supset(E z)\left(x=q_{d(e)}(z) \wedge(E y) R(n, z, y)\right)\right] \\
\leftrightarrow & q_{h(n)} \simeq q_{e} \leftrightarrow h(n) \in \theta q_{e} . \text { So } \alpha R_{1} \theta q_{e} .
\end{aligned}
$$

It is evident that a Gödel number of $h$ can be computed, uniformly in $e$ and a Gödel number of $R$, although we shall not use this fact. 
Lemma 3. For any e, the defining predicate of $\theta q_{e}$ can be written in $A E$ form in the arithmetic hierarchy.

ProOF.

$$
\begin{aligned}
n \in \theta q_{\odot} & \leftrightarrow(z)\left[(E y) T_{1}(e, z, y) \wedge(E y) T_{1}(n, z, y) \wedge U\left(\mu y T_{1}(e, z, y)\right)\right. \\
& =U\left(\mu y T_{1}(n, z, y)\right) \cdot \vee \cdot(y)\left(\neg T_{1}(e, z, y) \wedge \neg T_{1}(n, z, y)\right] \\
& \leftrightarrow(z)(E y)\left[T_{1}\left(e,(z)_{1},(y)_{1}\right) \wedge T_{1}\left(n,(z)_{1},(y)_{2}\right) \wedge U\left((y)_{1}\right)\right. \\
& \left.=U\left((y)_{2}\right) \cdot \vee \cdot \neg T_{1}\left(e,(z)_{1},(z)_{2}\right) \wedge \neg T_{1}\left(n,(z)_{1},(z)_{2}\right)\right]
\end{aligned}
$$

where the scope of the quantifiers is a recursive predicate.

Proof of THEOREM. Let $\varnothing$ denote the null function, and let $T_{0}$ be the isomorphism type containing $\theta \varnothing$. Let $T_{1}$ be the isomorphism type of $\theta f$ where $f$ is any finite nonvoid function; this is well defined by Lemma 1 . That $T_{0} \neq T_{1}$ follows from the fact that $(\theta \varnothing)^{\prime}$ is recursively enumerable while, as shown in [1], $(\theta f)^{\prime}$ is productive for any $f \neq \varnothing$. Since $(\theta \varnothing)^{\prime}$ is creative, it follows from [4] that degree $(\theta \varnothing)=$ degree $(\theta \varnothing)^{\prime}=0^{\prime}$. By an argument of [1] (given there for sets rather than functions), if $f$ is any finite function, degree $\theta f=0^{\prime}$. Now if $f$ and $g$ are any two functions with infinite domains, it follows from Lemmas 2 and 3 that $\theta f R_{1} \theta g$ and $\theta g R_{1} \theta f$, so that $\theta f \cong \theta g$. It also follows from those lemmas that these sets are of degree $0^{\prime \prime}$, so that if $T_{2}$ denotes their isomorphism type, $T_{2}$ is distinct from both $T_{0}$ and $T_{1}$.

It may further be noted that if, for a recursively enumerable (r.e.) set $\alpha, \theta \alpha$ denotes $\left\{n \mid \alpha=\omega_{n}=\right.$ range $\left.q_{n}\right\}$, then the reduction procedures of Lemmas 1 and 2 can (with minor modifications) be used to show that the isomorphism type of $\theta \alpha$ is uniquely determined by whether $\alpha$ is null, finite or infinite, and that in fact that three possible types of $\theta \alpha$ are exactly those of Theorem 1. In particular, all infinite r.e. sets, independently of their degree of unsolvability, have recursively isomorphic index sets.

2. In [2], it is shown that if $f, g$ are partial recursive functions neither of which extends the other, the pair of index sets $(\theta f, \theta g)$ possesses the property of inducing double creativity of any pair $(\alpha, \beta)$ of disjoint r.e. sets such that $\theta f \subseteq \alpha, \theta g \subseteq \beta$. It is then relevant to inquire how many such pairs of index sets there are, up to double isomorphism (i.e., simultaneous isomorphism under a single recursive permutation). This is answered by the theorem below.

Definition 1. A partial recursive function has type 0,1 or 2 according as its domain is null, finite or infinite. The type of $f$ will be denoted by $T(f)$.

Definition 2. Functions $f, g$ will be called comparable if one extends the other; otherwise, $f$ and $g$ are incomparable. 
Proposition 1. For any pair $f, g$ of partial recursive functions, there exist disjoint r.e. sets $\alpha$ and $\beta$ with $\theta f \subseteq \alpha, \theta g \subseteq \beta$ if and only if $f$ and $g$ are incomparable.

Proof. This is a special case of Theorems 4.1 and 4.2 of [1].

THEOREM 2. Let $f_{0}, f_{1}$ be incomparable functions. Then $\left(\theta f_{0}, \theta f_{1}\right)$ is doubly isomorphic to $\left(\theta g_{0}, \theta g_{1}\right)$ if and only if the following conditions hold:

(1) $T\left(f_{0}\right)=T\left(g_{0}\right)$ and $T\left(f_{1}\right)=T\left(g_{1}\right)$,

(2) $g_{0}$ and $g_{1}$ are incomparable.

Proof. By Proposition 1, the incomparability of $f_{0}$ and $f_{1}$ implies the existence of disjoint r.e. sets $\beta_{0}, \beta_{1}$ with $\theta f_{0} \subseteq \beta_{0}, \theta f_{1} \subseteq \beta_{1}$. To prove the necessity of the above conditions, assume that $\pi$ is a recursive permutation such that $\pi\left(\theta f_{0}\right)=\theta g_{0}$ and $\pi\left(\theta f_{1}\right)=\theta g_{1}$. Condition (1) then follows from Theorem 1. Moreover, $\theta g_{0}=\pi\left(\theta f_{0}\right) \subseteq \pi\left(\beta_{0}\right)$ and $\theta g_{1}$ $=\pi\left(\theta f_{1}\right) \subseteq \pi\left(\beta_{1}\right)$ where $\pi\left(\beta_{0}\right), \pi\left(\beta_{1}\right)$ are disjoint r.e. sets, which by Proposition 1 implies condition (2). To prove sufficiency, assume that $g_{0}, g_{1}$ are incomparable, $T\left(f_{0}\right)=T\left(g_{0}\right), T\left(f_{1}\right)=T\left(g_{1}\right)$. For $i=0,1$ let $h_{i}$ be the 1-1 recursive function which reduces $\theta f_{i}$ to $\theta g_{i}$ provided by the lemmas to Theorem 1 ; recall that, as noted above, these reductions have the property that for each $n, q_{h_{i}(n)}$ is comparable to $g_{i}$. Now define a 1-1 recursive function $h$ by

$$
\begin{aligned}
& q_{h(n)}(x) \simeq q_{h_{0}(n)}(x) \quad \text { if } n \in \beta_{0}, \\
& q_{h(n)}(x) \simeq q_{h_{1}(n)}(x) \quad \text { if } n \in \beta_{1}, \\
& q_{h(n)}(x) \text { undefined otherwise. }
\end{aligned}
$$

We note first that for $i=0,1$ we have

$$
n \in \theta f_{i} \rightarrow n \in \beta_{i} \rightarrow q_{h(n)} \simeq q_{h_{i}(n)},
$$

so that $h(n) \in \theta g_{i} \leftrightarrow h_{i}(n) \in \theta g_{i}$. But by choice of $h_{i}, n \in \theta f_{i} \leftrightarrow h_{i}(n)$ $\in \theta g_{i}$. Thus $n \in \theta f_{i} \rightarrow h(n) \in \theta g_{i}$.

Conversely, assume $h(n) \in \theta g_{i}$, i.e., $q_{h(n)} \simeq g_{i}$. We then observe the following:

(a) $n \in\left(\beta_{0} \cup \beta_{1}\right)^{\prime} \rightarrow q_{h(n)}=\varnothing \rightarrow g_{i}=\varnothing$, which contradicts the hypothesis that $g_{i}$ and $g_{1-i}$ are incomparable.

(b) $n \in \beta_{1-i} \rightarrow q_{h_{1-i}(n)} \simeq q_{h(n)} \simeq g_{i}$. But as noted above, $q_{h_{1-i}(n)}$ and thus $g_{i}$ is comparable to $g_{1-i}$, which again contradicts the hypothesis.

We thus deduce that $h(n) \in \theta g_{i} \rightarrow n \in \beta_{i}$. But $n \in \beta_{i} \rightarrow q_{h(n)} \simeq q_{h_{i}(n)}$, while $q_{h_{i}(n)} \simeq g_{i} \leftrightarrow n \in \theta f_{i}$. Together, these yield $h(n) \in \theta g_{i} \rightarrow n \in \theta f_{i}$.

We have thus shown that $\theta f_{0}=h^{-1}\left(\theta g_{0}\right)$ and $\theta f_{1}=h^{-1}\left(\theta g_{1}\right)$, i.e., that $h$ is a 1-1 reduction of the pair $\left(\theta f_{0}, \theta f_{1}\right)$ to $\left(\theta g_{0}, \theta g_{1}\right)$. By symmetry of the hypotheses, $\left(\theta g_{0}, \theta g_{1}\right)$ is 1-1 reducible to $\left(\theta f_{0}, \theta f_{1}\right)$. So by Smull- 
yan's generalization [7] of Myhill's theorem, $\left(\theta f_{0}, \theta f_{1}\right)$ is doubly isomorphic to $\left(\theta g_{0}, \theta g_{1}\right)$.

There are thus exactly three isomorphism types of pairs of index sets of incomparable functions, corresponding to pairs of functions of types $(1,1),(1,2)$ and $(2,2)$. The situation for pairs of comparable functions appears to be more complicated. We do not know conditions which are both necessary and sufficient for double isomorphism of such pairs of index sets.

\section{REFERENCES}

1. J. C. E. Dekker and J. Myhill, Some theorems on classes of recursively enumerable sets, Trans. Amer. Math. Soc. 89 (1958), 25-59.

2. L. S. Hay, On creative sets and indices of partial recursive functions, Trans. Amer. Math. Soc. 120 (1965), 359-367.

3. S. C. Kleene, Introduction to metamathematics, Van Nostrand, New York, 1952.

4. J. Myhill, Creative sets, Z. Math. Logik Grundlagen Math. 1 (1955), 97-108.

5. H. Rogers, Jr., Gödel numberings of partial recursive functions, J. Symbolic Logic 23 (1958), 331-341.

6. N. Shapiro, Degrees of computability, Trans. Amer. Math. Soc. 82 (1956), 281299.

7. R. Smullyan, Theory of formal systems, Annals of Mathematics Studies No. 47, Princeton Univ. Press, Princeton, N. J., 1961.

NoRThampton, Massachusetts 